\title{
First case of Zika virus spread through sexual contact is detected in UK
}

\author{
Anne Gulland
}

London

The United Kingdom's first case of Zika virus transmission through sexual contact has been detected, Public Health England has confirmed.

The case concerns a woman, who has since made a full recovery, infected by her partner, who had been on holiday in an area where the virus was active.

As of 30 November some 265 cases of the Zika virus have been diagnosed in the UK since 2015, of which 181 are confirmed. Seven of the 265 cases have been diagnosed in pregnant women. Most of the travellers infected with the virus-190-have picked it up while travelling in the Caribbean.

Public Health England has reiterated its advice to people travelling to areas where transmission of the virus is active, mainly the Americas. It says that men, regardless of symptoms, should use condoms for six months after returning from countries with a high or moderate risk of Zika infection. Women should avoid conception and should use condoms or other barrier methods for eight weeks after leaving the area.
Dilys Morgan, Zika incident director at Public Health England (PHE), said, "PHE's advice is based on the fact that our main concern is to avoid infection in pregnancy, in order to avoid risk to the unborn child." The virus has been linked to microcephaly in babies born to mothers infected with Zika, as well as to Guillain-Barré syndrome.

Jimmy Whitworth, professor of international public health at the London School of Hygiene and Tropical Medicine, said it was not unexpected that sexual transmission of the virus had been detected in the UK.

However, he added, "About 60 cases of sexual transmission of Zika have been reported worldwide, so we think this is quite rare ... Discovering just how common it is for the virus to be passed during sex by a man or woman is a key focus for Zika researchers."

On 18 November the World Health Organization declared that Zika virus was no longer a public health emergency of international concern but was still a "significant and enduring health challenge." 